\title{
SUR LA CHAETOTAXIE DE LA LARVE INFESTANTE
}

\section{DE SCHISTOSOMA MANSONI}

\author{
Par P. H. VERCAMMEN-GRANJEAN
}

Au cours de travaux biologiques menés au laboratoire médical de Costermansville (Province du Kivu, Congo belge), j'ai pu observer, le 10 août 1950 , sur le corps des cercaires de $S$. mansoni, une série de poils extrêmement fins et délicats.

Je m'empresse de dire que je ne suis pas le premier à avoir signalé la présence de poils sur des cercaires de schistosomes. Dans leur remarquable travail sur $S$. mansoni et $S$. hæmatobium (voir Annals of Tropical Medecine \& Parasitology, vol. XXVIII, 1934), Gordon, Davey et Peaston signalent, à la page 381, la présence de poils sur l'appendice caudal de $S$. mansoni. Ils mentionnent que c'est le $\mathrm{D}^{r}$ Vogel, de l'Institut de Médecine tropicale de Hambourg, qui en fit la première découverte. Cependant, ni les premiers, ni le second de ces auteurs ne donnent de précisions topographiques concernant la pilosité en quesțion.

Conditions d'observation. - J'ai réalisé toutes mes observations à l'aide d'un microscope de marque «Bauche and Lomb», de type "Recherche », équipé d'un objectif «Fluorite $98 \times$ et d'oculaires $15 \times$. Il convient de travailler dans un endroit obscur et de rechercher patiemment l'optimum de lumière. Des écrans de couleur peuvent aider éventuellement : soit qu'ils reposent la vue, soit qu'ils fassent varier l'acuité visuelle. Il est de première importance d'étudier sur le vivant, en milieu « sérum humain 》 frais, clair, propre et légèrement tiédi $\left(30^{\circ} \mathrm{C}\right.$.). Les poils se voient plus aisément sur des cercaires encore légèrement en mouvement, ear ils sont euxmêmes, dans ce cas, animés de mouvements inverses, en raison de la viscosité du milieu. Ce contraste de mouvements les rend forcément plus apparents.

Disposition pileuse. - La garniture pileuse du corps de la cercaire de $S$. mansoni est constante et symétrique ; elle fut établie par

Ann. de Parasitologie, t. XXVI, x 5-6. - 1951. 
l'observation méthodique de plus de cinquantes cercaires de $S$. mansoni. Notons que ces cercaires furent obtenues par infestation de Biomphalaria (Planorbis) stanleyi, à partir de selles de bilharziens.

Le nombre des poils est de vingt-quatre, soit six de part et d'autre de l'axe de symétrie du corps cercarien, tant ventralement que dorsalement.

Les lieux d'implantation sont papilliformes. En outre, deux papilles non pilifères se situent antéro-latéralement dans la dépression qui suit le renflement buccal (voir fig.).

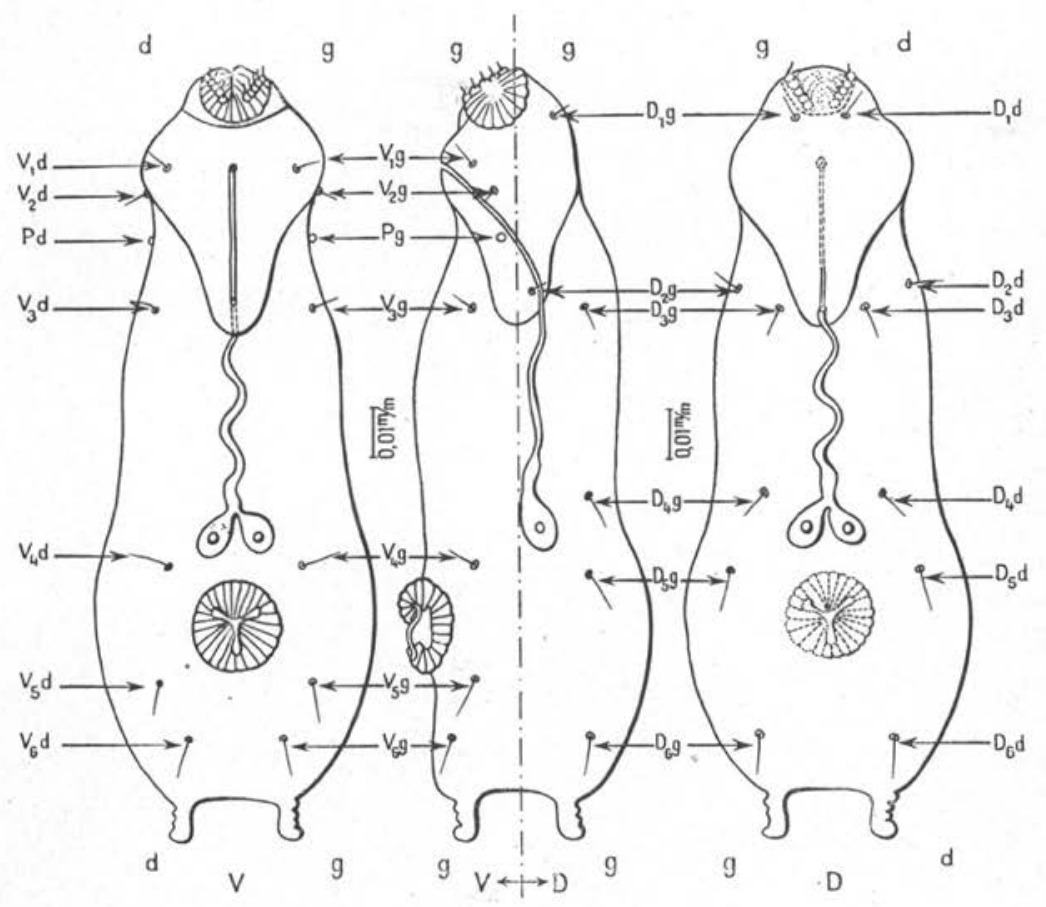

Fig. - Disposition des soies sur le corps de la cercaire de $S$. nansoni : $\mathrm{V}$ : face ventrale; D : face dorsale; $\mathrm{Vd}$ : soie ventrale droite; $\mathrm{Vg}$ : soie ventrale gauche; Dd : soie dorsale droite; Dg : soic dorsale gauche; $\mathrm{Pd}$ : papille droite; Pg: papille gauche.

Conclusion. - La découverte du système pileux du corps de la cercaire est, peut-être, une voie ouverte vers des possibilités systématiques nouvelles; cela pour autant que les cercaires très voisines 
de celle de $S$. mansoni, et telles que $S$. hæmatobium, $S$. bovis *, $S$. intercalatum, $S$. mattheei, etc..., aient des dispositions pileuses différentes.

J'ai tenté d'observer à mon tour les dispositions chætotaxiques sur la queue de la cercaire de $S$. mansoni. J'ai bien vu les poils signalés par Gordon, Davey et Peaston ; cependant, il m'a été impossible de relever la disposition caractéristique de ces poils, les spasmes caudaux étant par trop violents et persistant même après la mort de l'animal.

(*) Dans une lettre récente (31 octobre 1951), M. le I ${ }^{r}$ J. J. C. Buckley, Professeur à l'Ecole d'Hygiène et de Médecine. Tropicale de Londres. à qui j'avais fait part de mes travaux, confirma mes observations sur $S$. mansoni et me signala avoir pu observer également des poils sur le corps de la cercaire de $S$. bovis, sans autres données ou précisions topographiques.

(Laboratoire médical de Costermansville, province de Kiou, Congo belge) 